\title{
COMUNICACIÓN Y TIC. \\ Internet como factor de empoderamiento en estudiantes universitarios: actividades, relaciones, cooperación
}

\author{
Adrián Lucas López *
}

\section{Resumen}

El estudio indagó por los procesos de apropiación de Internet en las prácticas de un grupo de estudiantes universitarios de los distritos de San Miguel, Malvinas Argentinas, José C. Paz y Moreno del Distrito de Buenos Aires, Argentina; desde la perspectiva socio-histórica de Vigotsky, en la que involucró la observación conjunta de aspectos intelectuales, afectivo/emocionales y motivacionales y se tomó en consideración los niveles personal, relacional y colectivo que plantea la literatura sobre el empoderamiento.

Con base en entrevistas en profundidad y observaciones se encontró que la conexión aporta a la difusión y el intercambio de información, apoya el desarrollo académico, facilita la cooperación y permite que se adopten con mayor solidez y celeridad los códigos y prácticas propias del oficio de estudiante. La formación de comunidades y la disponibilidad de los dispositivos móviles promueven interacciones que aceleran la construcción de la afectividad.

Finalmente, las plataformas motorizan la realización de actividades en línea, sin embargo, la atención desmedida depositada en las pantallas es entendida como un obstáculo que desactiva la participación, la organización y la acción colectiva en los espacios físicos.

Palabras clave: apropiación de tecnologías, Internet, estudiantes, universitarios, empoderamiento, perspectiva socio-histórica.

\section{Recibido: Marzo 21, 2018 - Aceptado: Mayo 25, 2018}

* Licenciado en Comunicación por la Universidad Nacional de General Sarmiento (UNGS). Docente en UNGS y becario doctoral del Consejo Nacional de Investigaciones Científicas y Técnicas (CONICET) con sede de trabajo en el Instituto del Desarrollo Humano (IDH/UNGS). Juan María Gutiérrez 1150 (entre José León Suarez y Verdi) C.P. 1613 - Los Polvorines - Provincia de Buenos Aires - Argentina. Orcid. https://orcid.org/0000-0003-0645-6837.Email: Adrian.lopez1085@yahoo.com.ar 


\section{COMMUNICATION AND ICTS. \\ Internet as an empowerment factor in university students: activities, relationships, cooperation}

\section{Summary}

This study inquired about appropriation processes of the Internet in the activities of a university student group in the districts of San Miguel, Malvinas Argentinas, José C. Paz and Moreno of the District of Buenos Aires, Argentina, from Vygotsky's sociohistorical perspective, in which it was involved joint observation in terms of intellectual, affective / emotional and motivational aspects, and also, it is taken into consideration, personal, relational and collective levels provided by the literature about empowerment.

Based on in-depth interviews and observations, it was found that connectivity contributes to disseminate and exchange information, it supports academic development, facilitates cooperation and allows that the codes and interaction of being a student may be assumed with greater solidity and effectiveness. The emergence of communities and the availability of mobile devices promote interactions that increase affectivity construction.

Finally, platforms mobilize online activities, although the excessive attention paid on the screens is understood as an obstacle that disables participation, organization and collective action in physical spaces.

Keywords: appropriation of technologies, Internet, University, students, empowerment, Socio-historical perspective.

Received . March 21, 2018 - Accepted. May 25, 2018

\section{COMUNICAÇÃO E TIC. \\ Internet como fator de empoderamiento em estudantes universitários: atividades, relações, cooperação}

\section{Resumo}

O estudo investigou pelos processos de apropriação de Internet nas práticas dum grupo de estudantes universitários dos distritos de San Miguel, Malvinas Argentinas, José C. Paz e Moreno do Distrito de Buenos Aires, Argentina; desde a perspetiva sócio-histórica de Vigotsky, na qual envolveu a observação combinada de aspectos intelectuais, afetivos/ emocionais e motivacionais e tomou-se em consideração o níveis pessoal, relacional e coletivo que esboça a literatura sobre o empoderamiento.

Com base em entrevistas a fundo e observações foi achada a conexão que contribui à difusão e a troca de informação, apóia o desenvolvimento acadêmico, facilita a cooperação e permite que se adoptem com maior solidez e velocidade os códigos e práticas proprias da ocupação dos estudantes. A formação de comunidades e a disponibilidade dos dispositivos móveis promovem interações que apressam a construção da afetividade.

Finalmente, as plataformas motorizam a realização de atividades on-lines, porém, a atenção ilimitada depositada nas telas é compreendida como um obstáculo que incapacita a participação, a organização e a ação coletiva nos espaços físicos.

Palavras chaves: apropriação de tecnologias, Internet, estudantes, estudantes universitários, empoderamiento, perspectiva sócio-histórica.

Recebido. Março 21, 2018 - Aceitado. Maio 25, 2018 


\section{INTRODUCCIÓN}

La infraestructura comunicacional que se ha ido configurando en los últimos años ha tenido a Internet como tecnología privilegiada en torno a la cual se han alineado los medios y las empresas, los dispositivos de acceso, los contenidos culturales y los usuarios con sus prácticas. Un proceso signado por el avance acelerado de un conjunto de tecnologías digitales interactivas (TDI) en el que se destaca la incorporación y creciente penetración de los dispositivos móviles (hardware) junto con la proliferación de plataformas digitales multimedia (software). Dicha condición refuerza una clara tendencia hacia la profundización en el intercambio de información digital, la interacción social, el consumo de contenidos en nuevas pantallas y la posibilidad de los usos portables de la conexión.

Este contexto de trasformación técnica repercute sobre las relaciones sociales del conjunto de la población ya que los sujetos, de modos variados y complejos, atravesados por significativas desigualdades sociales, económicas y culturales, se ven afectados, ya sea por el contacto eventual con los artefactos como por la circulación de los discursos que tienen a la tecnología y sus funcionalidades como protagonistas.

El uso de las TDI suscita modificaciones a nivel colectivo que habilitan la ampliación en el acceso a la información y a la cultura, promoviendo derechos y libertades que mejoran la posición de las personas. Ahora bien, esas disposiciones son vivenciadas y significadas de múltiples modos por los diversos grupos sociales que las incorporan y las utilizan según sus propias coordenadas culturales.

En este marco se desarrolló el estudio que indagó los procesos de apropiación de Internet en las prácticas de un grupo de estudiantes universitarios, cuya dinámica es interrogada en lo relativo a su vinculación con los beneficios y acomodaciones que se producen a nivel personal, relacional y colectivo a partir del contacto con TDI.

Desde nuestra perspectiva, que toma como base el planteo sociohistórico de Vigotsky (1989) y su continuación en los estudios sobre la actividad en Leontiev (1983), los procesos de apropiación, para el caso de las TDI, comportan una instancia social de aprendizaje que implica la ejecución de actividades con artefactos técnicos o sus formas simbólicas. La misma se sitúa en un contexto sociocultural e histórico determinado en el que el sujeto participa a partir de condiciones desiguales, no solo en términos de acceso a los dispositivos, sino también en la construcción de las habilidades necesarias para la producción de mejoras en su calidad de vida.

De esta caracterización del fenómeno se desprende que en aquellos momentos del ciclo vital del sujeto en los que se producen vivencias [1] junto con TDI, estos sufren afectaciones de diverso tipo: a nivel intelectual, la persona adquiere saberes y competencias que le permiten reconstruir y actualizar sus conocimientos previos, como así también hacerse de nuevas habilidades para la realización de diversas prácticas y procedimientos; a nivel emocional, el sujeto no solo sufre una reconfiguración de su cognición, sino que también entabla una conexión afectiva y construye a lo largo del tiempo una disposición particular hacia los artefactos y los sentidos asociados a ellos; finalmente, también se producen modificaciones a nivel motivacional [2] debido a que el contacto con las diferentes tecnologías puede tener algún tipo de implicancia sobre el desarrollo de las acciones y la prosecución de las actividades, es decir, internet y sus recursos pueden funcionar como disparadores o vehículos primordiales de las acciones y permitir el desarrollo de tareas que de otro modo no podrían realizarse.

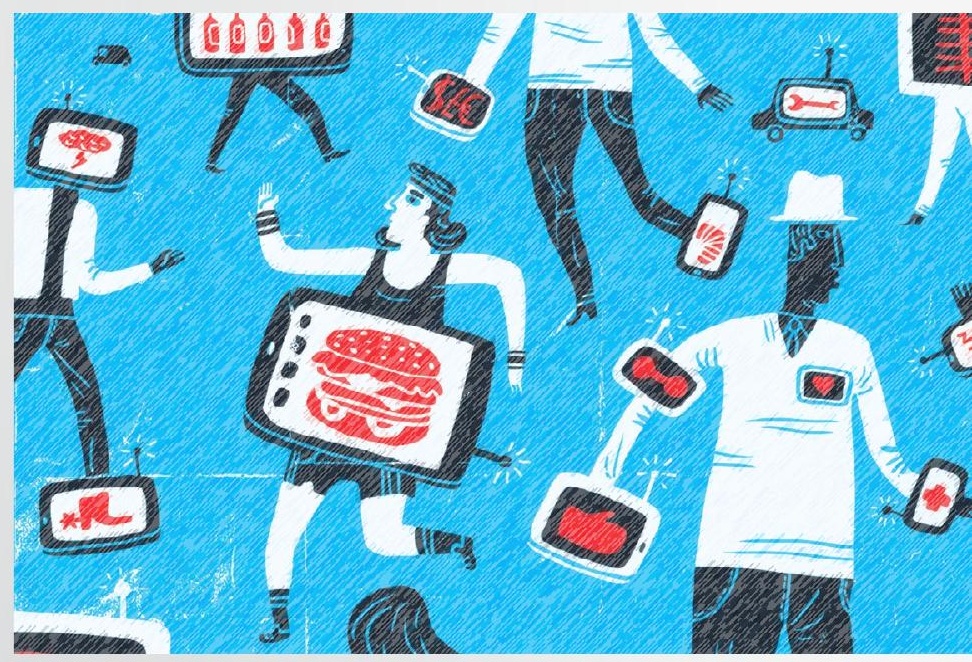

Por otro lado, la reflexión sobre la idea de "fortalecimiento", "potenciación" y posteriormente "empoderamiento", posee varias raíces en la literatura, provenientes de diversas disciplinas. Una de ellas es el enfoque de la Educación Popular desarrollado a partir del trabajo en los años 60 de Paulo Freire [3] , muy ligado a los 
trabajos de corte participativo desde la década de los 70. Otra base la componen los estudios dedicados a reflexionar sobre la situación de las mujeres y su condición desigual en los países periféricos, desde una mirada feminista focalizada en la perspectiva de género (Murguialday y otros, 2006; Rowlands, 1997). Y otro origen alternativo fue desarrollado por Julian Rappaport (1981; 1984; 1987) dentro del campo de la Psicología social comunitaria [4].

En la totalidad de estos trabajos se concibe una noción de empoderamiento asociada a un proceso en el que las personas, a partir de una toma de conciencia y la adquisición de nuevas capacidades, aumentan sus recursos cognitivos, afectivos y conductuales. Por tanto, lo que se ha denominado empoderamiento refiere a un proceso de reducción de la vulnerabilidad y de incremento de las propias capacidades que conduce a promover entre ellos un desarrollo humano sostenible.

Desde las distintas vertientes el concepto habilita su abordaje a partirdediferentes niveles deanálisis: se puede enfocar las modificaciones a nivel de lo personal, como el fortalecimiento del sí mismo, en términos de mayor confianza o adquisición de capacidades propias del individuo; se puede analizar la calidad de las relaciones sociales que mantienen los grupos y sus niveles de destreza para negociar, cooperar, influir e intercambiar conocimientos y recursos; y finalmente, es posible indagar lo colectivo asociado al afianzamiento de las identidades y estructuras políticas que le permiten a las agrupaciones obtener mayor poder de representación y avanzar hacia niveles superiores de influencia y acción colectiva.

\section{METODOLOGÍA}

El estudio fue desarrollado a partir de una metodología cualitativa. Se realizaron 34 entrevistas individuales en profundidad a estudiantes y se produjeron observaciones no estructuradas no participantes (Scribano, 2007) en el espacio de las universidades. Se respetaron las proporciones en que ambos sexos participan en la población estudiada y el grupo etario elegido fue de 18 a 35 años.

La estrategia seleccionada para el análisis fue el Método Comparativo Constante (Taylor, S. y Bodgan, R., 1987; Wimmer, M. y Dominick, J.,
1996) y se optó por un tipo de muestreo teórico propio de la teoría fundamentada. La población de estudiantes sobre la que se ciñe el estudio reside en la región novena, ubicada en el noroeste de la provincia de Buenos Aires. La misma comprende los distritos de San Miguel, Malvinas Argentinas, José C. Paz y Moreno, un espacio bastante desigual en términos habitacionales y en lo relativo a la situación del empleo. [5]

En esta área desarrollan sus actividades la Universidad Nacional de General Sarmiento (UNGS), la regional San Miguel de la Universidad Nacional de Lujan (UNLU), la Universidad Nacional de Moreno (UNM) y la Universidad Nacional de José C. Paz (UNPAZ). Los estudiantes que ingresan a las universidades de la región novena provienen, en una gran proporción, de sectores sociales desfavorecidos en términos de capital económico y cultural. Por diversas razones, asociadas a carencias económicas y a diversas dificultades en sus trayectorias educativas llegan a los estudios superiores con una considerable brecha entre sus propios conocimientos y las demandas académicas que requiere la universidad.

La mayoría de ellos trabajan para costear sus estudios, tienen a su cargo diversas responsabilidades familiares y son estudiantes de nuevo ingreso, que representan el primer miembro de la familia en continuar con los estudios superiores. Esta situación también incluye la gestión de las TDI que no involucra solamente a los distintos niveles de la educación formal previa, sino que se encuentra ligada al modo en el que los sujetos se encuentran insertos en el espacio social (Ezcurra, 2005, 2007, 2011; Gluz, 2011).

\section{ANÁLISIS Y RESULTADOS}

\subsection{SOBRE EL NIVEL PERSONAL [6]}

El análisis de los datos nos ha permitido visualizar cómo las actividades que realizan los estudiantes con los recursos de internet, en buena medida actividades de búsqueda, de interacción comunicativa y de producción de contenidos, a partir del asesoramiento que encuentran en la red, reposiciona a los sujetos en términos intelectuales. 


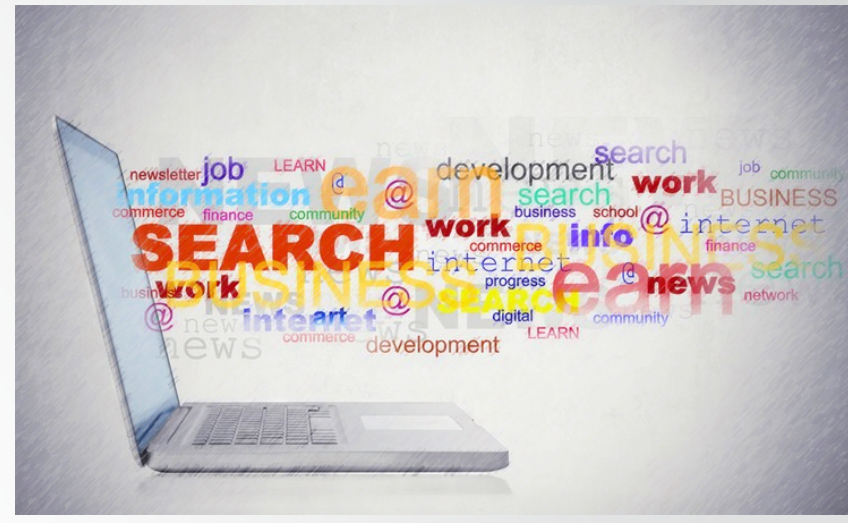

Desde el momento en que los estudiantes ingresan a la universidad se produce una ampliación de los usos de la conectividad. Se ejecutan nuevos procedimientos, se adquieren saberes y se participa en discusiones y prácticas productivas que los conducen a solucionar y afirmar diversos aspectos de su vida cotidiana y desempeño universitario.

La información que los estudiantes obtienen en la red facilita sus acciones y tareas de investigación y reflexión, además orienta el pensamiento y la toma de decisiones, no solo en relación a lo académico, sino también a lo administrativo o burocrático. particular hacia las TDI. En algunos casos, en los que ha habido un contacto sostenido con las herramientas, se ha identificado una ampliación de los saberes, acompañado de una sólida incorporación de los dispositivos, plataformas y recursos en las tareas y proyectos personales. Por el contrario, en otros casos, con una cantidad menor de exposiciones y con experiencias de menor profundidad en la construcción de conocimientos, la relación con los dispositivos y recursos tiende a la exterioridad o se significan negativamente, al asociar la intensidad de los usos con la pérdida de habilidades sociales en co-presencia.

A nivel emocional, los diferentes usos que comentan y sus representaciones sobre la circulación de la información describen una multiplicidad de matices. En algunos casos, esos saberes adquiridos junto con la conexión estimulan un apuntalamiento personal, que se traduce en una mayor previsión y capacidad para proyectar y planificar diversos aspectos de su desempeño práctico en el ámbito universitario.

El uso de las diferentes herramientas aumenta la confianza en la realización de las acciones
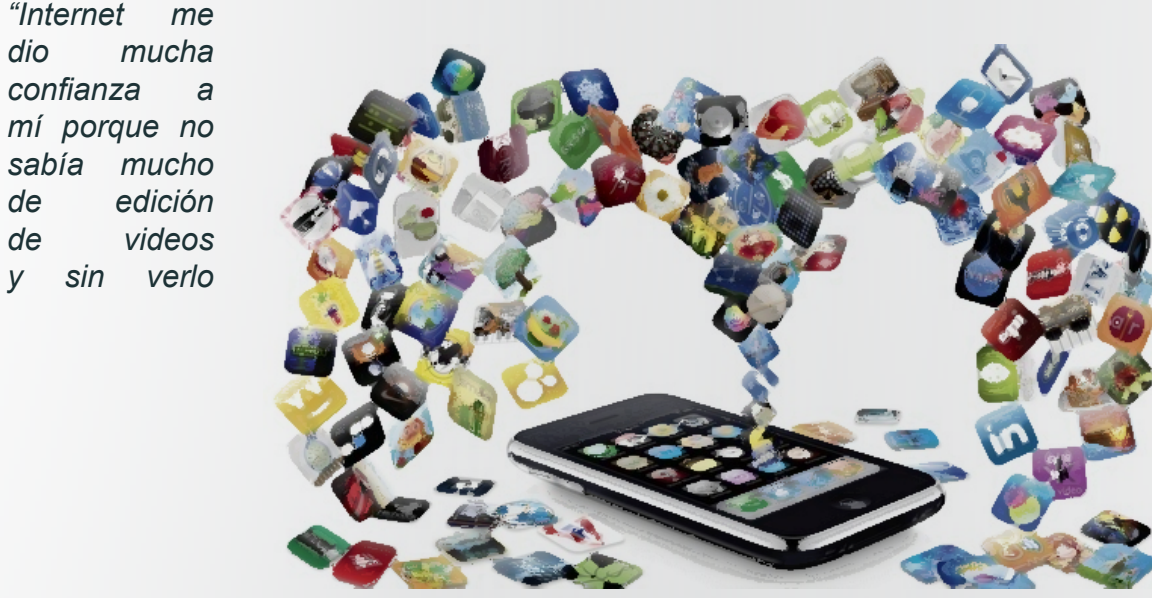

$y$ el sentimiento de integración personal, en el sentido de un mayor protagonismo en las actividades que corresponden con sus propios intereses, como también en relación a su participación en debates públicos y grupales, sumamente importantes para el período de la vida en el que se encuentran y para la actividad que están desarrollando.

académicamente hice toda una base de conocimiento manejándome con tutoriales de YouTube y empecé a editar videos sin tener el desarrollo de la materia Práctica 1"

(Ana, 26 años, estudiante de la Tecnicatura en producción de medios audiovisuales, UNPAZ).

Si bien estamos atentos en la observación a las múltiplesexperienciasy posiciones, lastrayectorias que comentan los estudiantes permiten resaltar la influencia de los conocimientos adquiridos a partir de sucesivas aproximaciones. Este factor es nodal y permite construir una disposición
Además de esta ampliación en términos culturales se valora significativamente la posibilidad del acercamiento y la conexión con los espacios y las personas:

Otras experiencias demuestran algunos temores y desconfianzas sobre la circulación de la información por la red y la posible peligrosidad de las interacciones y la exposición de las personas en las diferentes aplicaciones y servicios sociales. Asimismo, en algunos casos, la intensidad creciente de los usos de los dispositivos conectados de manera constante es asociada a la construcción de un vicio o hábito malicioso. 
Finalmente, en relación a lo motivacional las conversaciones mantenidas con los estudiantes nos permiten observar el mismo tipo de posiciones encontradas. Para algunos, las TDI promueven la realización de las tareas e impulsan el desarrollo de las prácticas ya que el uso de los recursos de la red es comprendido como una ampliación considerable en el horizonte de posibilidades.

En estos discursos, la conexión aparece como la herramienta primordial ante la resolución de los problemas e inquietudes y la ejecución de las actividades. Los recursos de internet son significados como amplificadores necesarios de la información, muy necesarios para transitar la vida y la producción en la universidad ya que promueven una toma de decisiones más avisada y producen un afianzamiento de las prácticas relativas a lo que se denomina oficio de estudiante (Coulon, 2008). Complementariamente, la universidad, en tanto lugar asociado con la producción de conocimiento formal y sistemático, pero también como espacio de encuentro y sociabilidad, aparece como orientadora de las búsquedas y los intereses en los estudiantes. Sin embargo, para otro grupo de estudiantes, la irrupción de la conexión y sus dispositivos en el espacio son entendidos como distracciones, usos nocivos que, dependiendo de su intensidad, pueden convertirse en "vicios". Según esta línea de pensamiento, la intensa y sostenida ubicación de la atención de los estudiantes en lo que sucede en la red hace que los mismos quieran establecer limitaciones en los usos.

En líneas generales, la intensidad de las prácticas, la cantidad de experiencias de construcción de conocimientos y la disposición elaborada a lo largo del tiempo hacia las TDI componen las únicas variables que dividen aguas. Los entrevistados que demuestran haber construido saberes en diversas oportunidades, ayudados por recursos de internet, que valoran este hecho y muestran una disposición positiva acorde con esta situación, no suelen inquietarse por aquellos supuestos perjuicios de la red y manejan, sin problemas aparentes, la distribución de las frecuencias e intensidades de los usos y ese punto en particular no les produce mayores preocupaciones.

\subsection{SOBRE EL NIVEL RELACIONAL [7]}

Las relaciones sociales que se producen en este tipo de universidades a partir de la utilización de los recursos de la red se han constituido como uno de los principales vehículos de intercambio de información y experiencias significativas entre los estudiantes.

Enlosdiversosservicios de redessociales digitales, de los cuales Whatsapp y Facebook son los más utilizados, los estudiantes forman agrupaciones por asignaturas o por carreras, y en esas redes más acotadas, los estudiantes mantienen buena parte de sus conexiones, comparten afinidades electivas y construyen afiliaciones. Allí circula información administrativa y una gran cantidad de datos académicos necesarios para la realización de las actividades, el estudio y los trabajos prácticos.

La evidencia nos permite aseverar que buena parte de la sociabilidad universitaria se ha trasladado al ámbito de las redes digitales. Sin embargo, se erigen distintas opiniones respecto de la eficiencia y el alcance de esas interacciones. Para algunos las relaciones a través de TDI involucran más ventajas que desventajas ya que valoran el beneficio del intercambio de información y experiencias y la disponibilidad constante de las personas, pero también están aquellos que ven en las interacciones en línea, una zona muy limitada a un cierto tipo de interacciones comunicativas, con determinadas intenciones, dadas las limitaciones expresivas de las plataformas. Este tipo de consideraciones conduce a que los estudiantes refuercen la interacción digital con encuentros cara a cara planificados:

"Alguna experiencia de hacer un trabajo en grupo. A veces puede ser conflictivo, porque lo que se pierde es la cosa de mirarte a la cara y quizás algo que decís, no sabes cómo se recibe el mensaje, emocionalmente. Si yo te veo a vos ahora y me parece una pelotudez lo que estás diciendo, trataría de que no parezca que es una pelotudez. Cuando lo escribo o cuando me agarra caliente y bueno sí, está ese riesgo. Porque capaz no se entiende o se malinterpreta."

(Nicolás, 26 años, estudiante de Trabajo social, UNPAZ). 
Con respecto al carácter emocional de las relaciones se ha podido observar que este tipo de intercambios constantes y sostenidos en el tiempo afianza los lazos afectivos generados en co-presencia, y en algunos casos, produce relaciones sólidas y duraderas que trascienden el vínculo universitario.

Estas ligazones, que se mantienen en el derrotero de conversaciones continuas e incesantes, se cristalizan debido a la temprana visibilidad de aspectos de la vida privada que son distribuidos por los estudiantes en los servicios de redes sociales digitales. Existen casos en los que estas relaciones sostienen el desarrollo de la vida universitaria en diversos aspectos y permiten la comprensión de códigos y costumbres, así como acompañan emocionalmente y refuerzan el tránsito por la vida universitaria. Sin embargo, también existen aquellos que las comprenden como espacios restrictivos, al haber experimentado serias discusiones, originadas por malentendidos y confusiones, derivadas del ambiente comunicativo en las plataformas digitales.

En relación a lo motivacional es importante destacar que, a pesar de que se verifican ambas posiciones, son ampliamente valoradas las facilidades obtenidas por el uso de las herramientas portables de comunicación en línea. Estos nuevos lazos reticulares que trascienden las paredes universitarias habilitan una gran cantidad de acciones y desempeños e impulsan la coordinación para el trabajo académico, que luego se puede complementar con encuentros en co-presencia.

Las interacciones y los intercambios que habilitan las herramientas digitales son valoradas por los estudiantes y se capitalizan como un recurso necesario e indispensable para la vida universitaria, sin embargo este tipo de conexiones encuentran las limitaciones propias del soporte comunicativo.

\subsection{SOBRE EL NIVEL COLECTIVO [8]}

Algunos estudiantes consideran que el uso de TDI en las universidades estudiadas permite la consolidación de grupos que se conectan intersubjetivamente, se reconocen como parte del colectivo y, a lo largo del tiempo, se afianzan con más fuerza, adquiriendo así mayores niveles de fortaleza interna y representación externa. Al participar en estos grupos, los estudiantes toman contacto con los discursos y saberes que circulan en la universidad, los temas, los problemas y las discusiones propias de ese espacio, lo cual es importante en términos de socialización.

En aquellas plataformas tienen la posibilidad de lograr una mayor visibilidad de cara al conjunto de la comunidad educativa y un nivel considerable de representación política, en tanto colectivo interesado en difundir ideas y proponer consignas. Los estudiantes se agrupan a partir de diversos

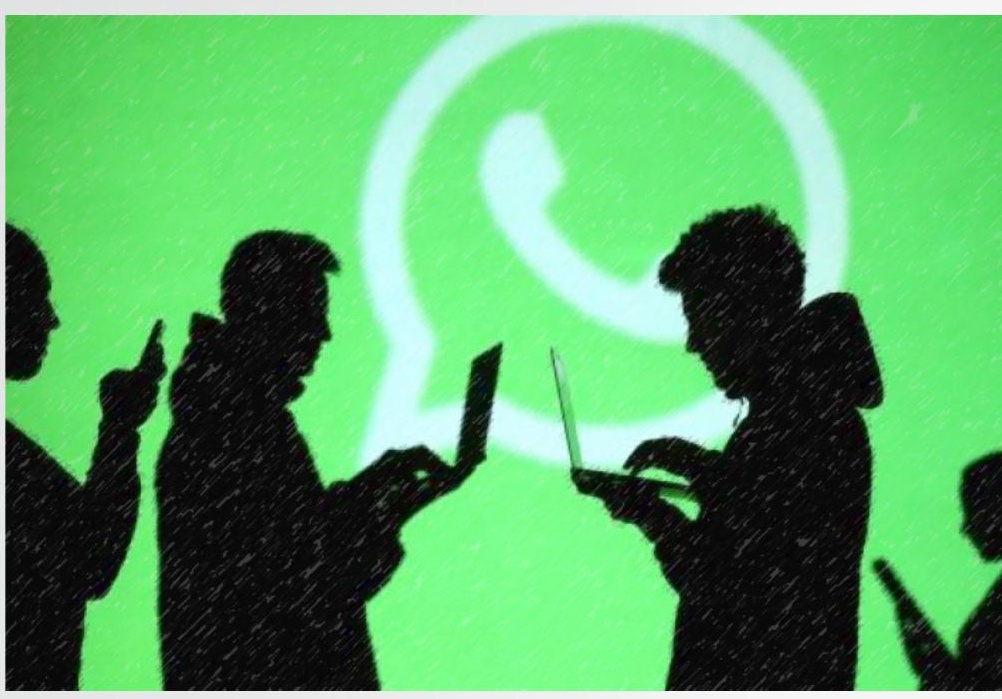

objetivos y con diferentes alcances, como pueden ser los asociados a las asignaturas que cursan, los relativos a las diferentes carreras o aquellos vinculados con diferentes agrupaciones políticas. En estos colectivos, distribuyen opiniones, pensamientos, además, discuten, intercambian, reflexionan y establecen la reproducción ampliada de las formas de sociabilidad que se inauguran en los espacios físicos de las universidades.

Por otra parte, las relaciones se solidifican de modo tal que se producen situaciones de cooperación entre sus participantes. Ante alguna evidencia de posibles dificultades para el desarrollo de las tareas que solicita el trabajo académico, las entregas de los ejercicios prácticos o hasta la propia continuidad de la cursada de las diferentes asignaturas, los estudiantes despliegan tácticas persuasivas de comunicación para lograr que no se produzcan las faltas, las fallas o el desgranamiento. La interacción virtual promueve la identificación temprana y afianza las relaciones y vivencias compartidas.

"Si un compañero pasó la información a otro que quiere dejar, se lo incita a que no deje. Después la discusión se da cara a cara, pero lo primero es 
mandarle un WhatsApp para ver dónde está para ir a verlo y apoyarlo para que no deje." (Andrés, 35 años, estudiante de Trabajo social, UNM)

Finalmente, se ha registrado una posición crítica con respecto a la presencia de las TDI en el entorno de la universidad, que considera que la atención de los estudiantes sobre las pantallas de los diferentes dispositivos móviles y computadoras portátiles puede perjudicar el diálogo fluido y necesario entre los estudiantes o el involucramiento en las prácticas significativas que se desarrollan en el espacio físico de la universidad. Desde esta perspectiva, las pantallas conectadas resultan una suerte de distracción contraproducente y una inhibición para el desarrollo de las actividades y el involucramiento político.

"Internet mejoró mucho el modo de comunicarnos, te lo digo desde la agrupación, porque es desde donde más lo puedo reflejar, como pasar la información a los compañeros, nosotros avisamos que llegaron los cheques para las becas, o sea en comunicación, es positivo, ahora nos pasa que como ya se enteraron, nosotros a lo mejor estamos abajo y no pasan, antes quizá, '¿nos tomamos un mate?'(...) ahora ya se enteraron por el face, entonces es entrar a cursar o entrar a buscar el cheque, en ese sentido lo veo negativo (...) hay un impacto en el tiempo que uno le dedica al otro. (...) incluso perdés la mirada, cuando entras a la universidad entrás mirando el celular, perdés la oportunidad de relacionarte desde otro lado con el colectivo universitario, me parece que eso también se perdió (...) termina siendo un depósito, entramos, estudiamos y salimos, dejan de darse ese tipo de relaciones que se daban cuando poníamos un poquito la cara y no la teníamos inmersa en el dispositivo.

(...) imagino yo, antes cuando no estaba el acceso tan fuerte a lo que pasa afuera, porque estar acá y con esto (apunta con la mano el celular) en realidad estás en lo que pasa afuera, ya sea tu casa, tu trabajo, o sea, creo que se perdió hasta la posibilidad de vivir lo que estás haciendo, por ejemplo, estás acá hoy en la universidad, pero estás con la cabeza en que dejaste a tu hija haciendo tal cosa o que en tu trabajo está pasando tal otra o sea que se perdió eso de estar completamente en mente y cuerpo, todo en el mismo lugar. (...) Por eso se hace tanto hincapié desde las agrupaciones a esto de hagamos asambleas, creo que tratamos de estar en espacios donde podamos dialogar y debatir y eso creo que es lo que se va a perder en la medida que esto siga creciendo, si no le damos mucho hincapié a esto a, juntémonos, organicémonos, incluso si esto continua en el tiempo, creciendo, hasta puede dejar de ser necesario el centro de estudiantes para el grueso de los universitarios."

(Natalia, 35 años, estudiante de Trabajo social, UNLU)
Los discursos sobre la influencia de la conectividad y los servicios de las plataformas digitales en la gestión de lo colectivo proponen planteos contrapuestos. Por un lado, algunos consideran que los servicios digitales contribuyen con la consolidación de los colectivos estudiantiles, al posibilitar más y mejores vinculaciones entre los participantes y mayor visibilidad de cara a la comunidad educativa, pero por otro lado, se encuentran aquellos que comprenden la utilización de las herramientas en el espacio físico de la universidad como una distracción generalizada para el estudiantado y un modo de desactivar la acción conjunta y organizada.

\section{CONCLUSIONES}

El análisis realizado nos permite aseverar que, en el grupo de estudiantes de la región observada, las apropiaciones de internet que se realizan contribuyen con la formulación de un tipo de socialización universitaria más sólida y veloz. A pesar de las limitaciones expresivas de la estructura, la integración en las diferentes plataformas aporta a la difusión y el intercambio, apoya el desarrollo académico y facilita la cooperación, lo cual permite que se adopten con mayor celeridad los códigos, sentidos y prácticas que construyen el oficio del estudiante (Coulon, 2008).

Por otro lado, la formación de comunidades y la disponibilidad de los dispositivos móviles y portables permiten la extensión del mundo universitario (temas, problemas, sujetos y relaciones) a la multiplicidad de espacios superpuestos que componen la vida cotidiana $y$, a su vez, las preocupaciones y cuestiones de la dinámica diaria y el trabajo ingresan con mucha más facilidad al espacio de la universidad.

Este proceso, de combinación difusa entre los momentos dedicados a la producción formal y sistemática y los tiempos consagrados a la diversión y el disfrute (Igarza, 2009), en el espacio universitario, promueve interacciones que aceleran la construcción de la afectividad. La sociabilidad en las plataformas, fortalece los lazos iniciados en la co-presencia y acerca los mundos privados y afectivos de los estudiantes que componen relaciones de amistad más profundas en menos tiempo. 


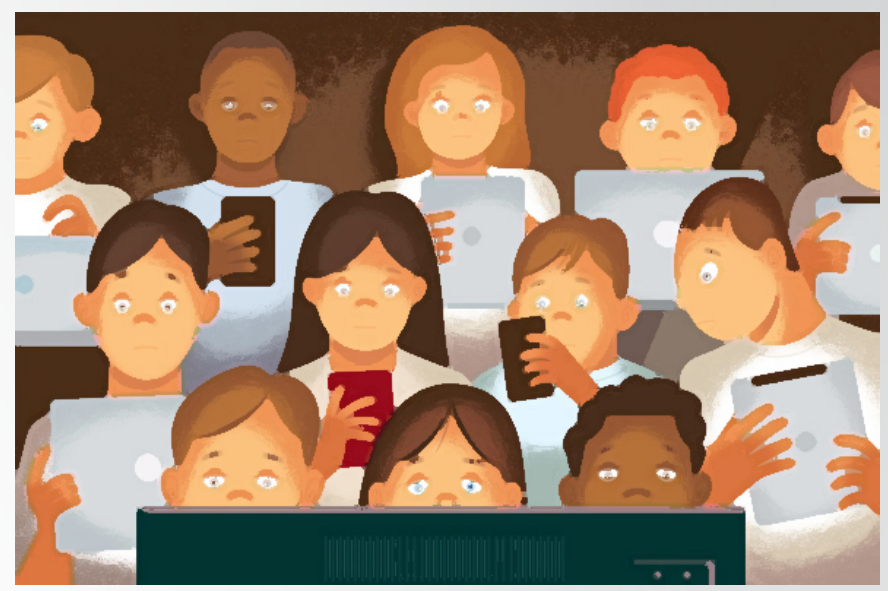

Finalmente,enrelaciónconelaspectomotivacional, se ponen en juego las consideraciones sobre el plano virtual y el terreno de lo presencial. Si bien los estudiantes sostienen que la apropiación de las TDI motoriza la realización de actividades, el intercambio y la comunicación en línea, las relaciones en el espacio se ven perjudicadas debido a la atención desmedida sobre las pantallas que anula los cuerpos, necesarios para el compromiso y la acción política.

\section{NOTAS}

[1] En este punto es importante aclarar que lo vivencial desde la teoría socio-histórica hace referencia a la experiencia específica de algún suceso siempre externo al sujeto y que se produce a partir de la conjunción entre la personalidad de los participantes y el medio o entorno material y simbólico. La personalidad desde esta mirada tiene en cuenta tanto aspectos cognitivos como emocionales (Del Cueto, 2015, p.34)

[2] En este estudio comprendemos lo motivacional desde la teoría de la actividad de Leontiev que se encuentra en la base de nuestra conceptualización sobre los procesos de apropiación. Para este autor los motivos refieren a aquellos "motores" que impulsan y orientan la acción. Esto es, construcciones de sentido que actúan como razones subyacentes de la acción que impulsan y conducen los comportamientos voluntarios. Sin embargo, estos motivos, en tanto necesidades como fuerza interior, no son previos a la ejecución de las actividades, sino que se producen en la relación que establece el sujeto con el entorno y a través de las múltiples mediaciones que mantiene con objetos y signos. Si bien el autor reconoce que el hombre nace dotado de necesidades vitales propias de lo orgánico, y aquí las necesidades y deseos anteceden a la acción, ni bien el sujeto empieza a actuar se construyen motivaciones, más complejas y de orden simbólico, que promueven las actividades (Leontiev, 1984, pp.147-161).

[3] En este autor es primordial la noción de diálogo como un componente central de su pedagogía orientada a la liberación (Freire, 2008). Esta perspectiva, avisada sobre la opresión y las condiciones desiguales que sufren los sujetos en las sociedades capitalistas actuales, apunta la necesidad de desarrollar acciones que les permitan a todas las personas participar en la construcción de su entorno social y cultural.

[4] Al interior de la Psicología general, la Psicología Social-Comunitaria es un subcampo o ámbito de investigación cuyo objeto de estudio son las dificultades, carencias o conflictos de los grupos - comunidades a partir de factores sociales, culturales y/o ambientales. En un contexto de desigualdad y poca influencia del Estado en el desarrollo social, este enfoque reflexiona sobre posibles formas de intervención, orientadas al mejoramiento de las condiciones de vida de la población a partir del fortalecimiento comunitario.

[5] El último Censo Nacional de Población realizado en 2010 reveló que dicha región cuenta con una población total de 1.316.951 habitantes. Del total de hogares de la región (365.703) un $84.39 \%$ no tiene acceso a red cloacal y un $34 \%$ habita viviendas con pisos de cemento, ladrillo o tierra. Según resultados de la Encuesta Permanente de Hogares, durante el primer semestre de 2017 el $32.6 \%$ de las personas residentes en el Conurbano Bonaerense viven bajo la línea de pobreza y el $8.2 \%$ bajo la línea de indigencia. En lo que respecta al empleo, en el tercer trimestre de 2017 la tasa de desocupación era del $10.3 \%$ y la de subocupación del $11.8 \%$.La penetración de TDI es significativa. De acuerdo con los resultados del Censo 2010 , el $41 \%$ de los hogares de la región poseen computadora, el $88 \%$ posee teléfono celular y el $49 \%$ posee telefonía de línea fija. La última Encuesta Nacional sobre Acceso y Uso de Tecnologías de la Información y la Comunicación (ENTIC) con resultados del período de mayo-julio de 2015 arroja que el $87,3 \%$ de los hogares urbanos de los partidos del Conurbano Bonaerense dispone de teléfono celular, el $63,1 \%$ posee algún tipo de computadora y el $57,2 \%$ tiene acceso a Internet. 
[6] El nivel personal focaliza sobre el registro de aquellos rasgos y cualidades del estudiante que se ven trastocados por el contacto con TDI, las repercusiones que se producen sobre las actividades que desarrolla, las valoraciones que hace al respecto y el tipo de disposición que demuestra haber construido hacia el uso de los recursos de la conexión y hacia los dispositivos que la permiten.

[7] La observación de lo relacional implica trascender la atribución de variables sobre sujetos, requiere que ubiquemos la categorización sobre las formas de las relaciones, sus dinámicas y el tipo de interacciones entre individuos que producen relaciones y redes sociales. Para algunos autores como Bott (1990) el sujeto no compone estrictamente un grupo, sino es mediante su participación en una configuración de relaciones personales, esto es, una red que lo integra a ese mundo simbólico y lo posiciona de otro modo en su entorno. En este proceso el sujeto se encuentra constantemente ejerciendo negociaciones de sentido con los otros integrantes del grupo sobre la calidad de las relaciones y los recursos resultantes. Todas estas características se extienden para las redes establecidas mediante dispositivos digitales (Piscitelli, 1995; Castañeda et al, 2011).

[8] La conformación de redes sociales a partir de las relaciones e interacciones entre los participantes estructura y orienta la formación más o menos estable de grupos emergentes. En este punto es posible establecer una relación con el concepto de capital social. Para Bourdieu (1986) las personas conforman tramas de relaciones de las cuales obtienen recursos, afectos o algún tipo de información. Estas redes, muchas veces logran estabilizarse como un núcleo duro compuesto por un número reducido de personas con las que se mantiene una relación relativamente perdurable. Ahora bien, para el caso de nuestro estudio nos interesa observar el nivel de consolidación de esos agrupamientos y el modo en el que repercuten esas formaciones en las prácticas académicas y en la sociabilidad universitaria.

\section{REFERENCIAS}

- Bott, E. (1990). Familia y red social. Roles, normas y relaciones externas en las familias urbanas corrientes. Madrid: Ed. Altea.

- Bourdieu, P. (1986). The forms of Capital. In Richardson J. G. (edit.) Handbook of theory and research for the sociology of education (pp. 240-268). New York: Greenwood.

- Castañeda, L.; González, V. \& Serrano, J.L. (2011). Donde habitan los jóvenes: precisiones sobre un mundo de redes sociales. In Martínez, F. y solano, I. (Eds.), Comunicación y relaciones sociales de los jóvenes en la red. (pp. 47-63). Alicante: Marfil.

- Coulon, A. (2008). A condiçao de estudante: a entrada na vida universitária, Salvador de bahía: Edufba

- Del Cueto J. D. (2015). Dos nociones para un enfoque no escisionista de las emociones y la afectividad: Situación social del desarrollo y vivencia en Vigotsky. [Two notions for a non-divisive approach to emotions and affectivity: Social situation of development and experience in Vygotsky] Perspectivas en psicología, 12, 29-35. https://bit.ly/2s7Evl7

- Ezcurra A. M. (2011). Igualdad en educación superior: Un desafío mundial, Los Polvorines: Universidad Nacional de General Sarmiento, Instituto de Estudios y Capacitación, Federación Nacional de Docentes Universitarios.

- Ezcurra A.M. (2007). Los estudiantes de nuevo ingreso: democratización y responsabilidad de las instituciones universitarias. Universidad de San Pablo: Cuadernos de Pedagogía Universitaria.

- Ezcurra, A. M. (2005). Diagnóstico preliminar de las dificultades de los alumnos de primer ingreso a la Educación Superior. [Preliminary diagnosis of the difficulties of students entering first year of Higher Education] Perfiles Educativos, 107 118-133. https://bit.ly/2s7RCCK 
- Felice, Magdalena (2013). PERFILES DE USUARIO: Usos y Apropiaciones del Teléfono Celular en Jóvenes de la Ciudad de Buenos Aires. Revista Luciérnaga, Año 5, N9. Grupo de Investigación en Comunicación, Facultad de Comunicación Audiovisual, Politécnico Colombiano Jaime Isaza Cadavid. Medellín-Colombia. ISSN 2027-1557. Págs 18-28. Disponible en: http://www.politecnicojic.edu.co/ images/downloads/publicaciones/revistaluciernaga/luciernaga-09/pdf/usos_ perfiles_espanol(si).pdf

- Freire, P. (2008). Pedagogía del oprimido. Buenos Aires: Siglo XXI.

- Gluz, N. (Edit.) (2011). Admisión a la universidad y selectividad social: cuando la democratización es más que un problema de "ingresos", Los Polvorines: Universidad Nacional de General Sarmiento.

- Igarza, R. (2009). Burbujas de ocio. Nuevas formas de consumo cultural. Buenos Aires: La Crujía.

- Leontiev, A (1983). El desarrollo del psiquismo, Madrid: Akal

- Leontiev, A. (1984). Actividad, conciencia y personalidad, México D.F: Editorial Cartago.

- Murguialday, C., de Armiño, K. y Eizagirre, M. (2006) Empoderamiento, In Diccionario de Acción Humanitaria y Cooperación al Desarrollo, Universidad del País Vasco. https://bit.ly/1B8WZUa

- Psicitelli, A. (1995). Enredados. Ciudadanos de la cibercultura. In Dabas E. y Najmanovich, D. (Comp.), Redes. El lenguaje de los vínculos hacia la reconstrucción y el fortalecimiento de la sociedad civil. (pp. 77-103) Buenos Aires: Paidós.

- Rappaport, J. (1981). In praise of paradox: A social policy of empowerment over prevention. American Journal of Community Psychology, 9, 1-21. https:// bit.ly/2kj4CBX

- Rappaport, J. (1984). Studies in empowerment: Introduction to the issue. Prevention in Human Services, 3, 1-7. https://doi.org/10.1300/J293v03n02_02

- Rappaport, J. (1987). Terms of Empowerment/Examples of Prevention: Toward a theory for community psychology. American Journal of Community Pshychology, 15 (2), 121-148. https://bit. $\mathrm{ly} / 2 \mathrm{~s} 5 \mathrm{Vc0Q}$
- Rowlands, J. (1997). Questioning Empowerment, Oxford: Oxfam

- Scribano A. O. (2007). El proceso de investigación social cualitativo, Buenos Aires: Prometeo

- Taylor, S. y Bodgan, R. (1987). Introducción a los métodos cualitativos de investigación, Buenos Aires: Paidós.

- Vigotsky, L. (1989). El desarrollo de los procesos psicológicos superiores, Barcelona: Crítica.

- Wimmer, M. y Dominick, J., (1996). La investigación científica de los medios de comunicación. Una introducción a sus métodos, Barcelona: Bosch Casa editorial S.A.

\section{Para citar este artículo:}

$$
\text { Lucas - López, A. (2018). }
$$

COMUNICACIÓN Y TIC. Apropiación de Internet como factor de empoderamiento en estudiantes universitarios: actividades, relaciones, cooperación. Revista Luciérnaga / Comunicación. Año 10, N19. Págs. 71-81.

OJS. http://revistas.elpoli.edu.co/index.php/luc/issue/archive Link. http://www.politecnicojic.edu.co/index.php/revista-luciernaga 